\title{
Highly Phase Separated Aromatic Ionomers Bearing Perfluorosulfonic Acids by Bottom-up Synthesis: Effect of Cation on Membrane Morphology and Functional Properties
}

Olesia Danyliv, Cristina Iojoiu, Sandrine Lyonnard, Nicolas Sergent, Emilie Planes and Jean-Yves Sanchez

Table SI1. Summary of demethylation reactions performed on the intermediate $\mathbf{3}$ in order to obtain the monomer DHSPFS

\begin{tabular}{|c|c|c|c|c|}
\hline $\begin{array}{l}\text { Product upon } \\
\text { demethylation }\end{array}$ & Reactant & Solvent & $\begin{array}{l}\text { Tempera- } \\
\text { ture, }{ }^{\circ} \mathrm{C}\end{array}$ & Observations \\
\hline \multirow{4}{*}{ Intermediate $\mathbf{3}$} & $\begin{array}{c}\text { Hydrobromic } \\
\text { acid }\end{array}$ & Acetic acid & $80^{\circ} \mathrm{C}$ & - formation of side products \\
\hline & Iodocyclohexane & Dimethylformamide & $150^{\circ} \mathrm{C}$ & $\begin{array}{l}\text { - 20-fold excess of the reactant does } \\
\text { not lead to the total demethylation } \\
\text { - formation of side products }\end{array}$ \\
\hline & Boron tribromide & $\begin{array}{c}\text { Acetonitrile / } \\
\text { dichloromethane }\end{array}$ & $50-80{ }^{\circ} \mathrm{C}$ & $\begin{array}{c}\text { - very long reaction (more than a } \\
\text { week) and does not lead to the total } \\
\text { demethylation }\end{array}$ \\
\hline & $\begin{array}{c}\text { Boron tribromide } \\
\text { / thiourea }\end{array}$ & $\begin{array}{c}\text { Acetonitrile / } \\
\text { dichloromethane }\end{array}$ & $50-80{ }^{\circ} \mathrm{C}$ & $\begin{array}{c}\cdot 20 \mathrm{~h} \text { at } 80^{\circ} \mathrm{C} \\
\text { - demethylated product is received } \\
\text { for batches of } 1 \mathrm{~g} \text { of the } \\
\text { intermediate } \mathbf{3} \\
\text { - demethylation does not proceed till } \\
\text { the end for batches of }>5 \mathrm{~g} \\
\text { - difficulties in purifying a } \\
\text { demethylated product }\end{array}$ \\
\hline $\begin{array}{l}\text { Intermediate } 3 \\
\text { in } \\
\text { tetrabutylam- } \\
\text { monium form }\end{array}$ & Boron tribromide & Dichloromethane & $25^{\circ} \mathrm{C}$ & $\begin{array}{l}\text { - successful dymethylation in 2-3 } \mathrm{h} \\
\text { - difficulties in transforming the } \\
\text { final product (DHSPFS) or the } \\
\text { ionomer }(\mathrm{P} 1-1.4) \text { in } \mathrm{K}^{+} \text {-form }\end{array}$ \\
\hline Intermediate 4 & Boron tribromide & Dichloromethane & $25^{\circ} \mathrm{C}$ & $\begin{array}{c}\text { - successful dymethylation in } 2-3 \mathrm{~h} \\
\text { - easy purification } \\
\text { - easiness in transforming DHSPFS } \\
\text { in its } \mathrm{K}^{+} \text {-form }\end{array}$ \\
\hline
\end{tabular}


Table SI2. Analysis of the P1 ionomers' degradation

\begin{tabular}{|c|c|c|c|c|c|c|}
\hline $\mathrm{M}^{+}$-form & Ionomer & $\begin{array}{l}\text { Thermal } \\
\text { degradation } \\
\text { onset, }{ }^{\circ} \mathrm{C}\end{array}$ & $\begin{array}{c}\text { Thermal } \\
\text { degradation } \\
\text { endset, }{ }^{\circ} \mathrm{C}\end{array}$ & $\begin{array}{c}\text { Relative } \\
\text { weight loss, } \\
\%\end{array}$ & $\begin{array}{l}\text { Molecular } \\
\text { weight loss, } \\
\mathrm{g} / \mathrm{mol}\end{array}$ & $\begin{array}{c}\text { Molecular } \\
\text { weight of S- } \\
\text { PFSA-M }{ }^{+} \text {side } \\
\text { chain, g/mol }\end{array}$ \\
\hline \multirow{3}{*}{$\mathrm{K}^{+}$-form } & P1-1.0 & 310 & \multirow{3}{*}{450} & 31.5 & 327.0 & \multirow{3}{*}{367.3} \\
\hline & P1-1.2 & 330 & & 35.6 & 310.2 & \\
\hline & P1-1.4 & 390 & & 38.1 & 293.5 & \\
\hline \multirow{3}{*}{$\mathrm{Li}^{+}$-form } & P1-1.0 & & & 22.0 & 221.3 & \multirow{3}{*}{335.1} \\
\hline & P1-1.2 & 250 & 430 & 25.0 & 209.8 & \\
\hline & P1-1.4 & & & 31.0 & 237.6 & \\
\hline \multirow{3}{*}{$\mathrm{H}^{+}$-form } & P1-1.0 & & & 25.0 & 250.0 & \multirow{3}{*}{329.2} \\
\hline & $\mathrm{P} 1-1.2$ & 130 & 300 & 28.8 & 240.0 & \\
\hline & P1-1.4 & & & 33.9 & 248.2 & \\
\hline
\end{tabular}

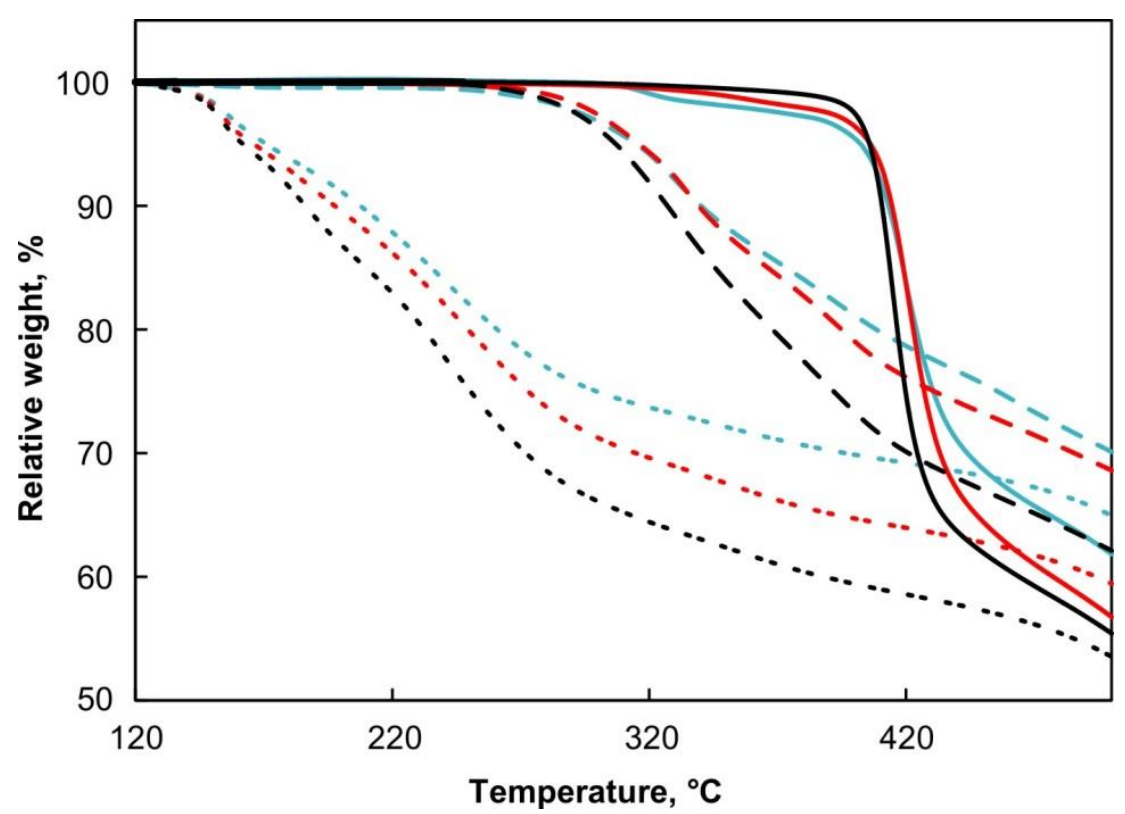

Figure SI1. Thermogravimetric curves of the P1 series of ionomers: blue curves represent P1-1.0 membranes, red curves - P1-1.2 samples and black curves - P1-1.4 membranes. Solid lines show the behavior of the ionomers in $\mathrm{K}^{+}$form, dashed lines - of the ionomers in $\mathrm{Li}^{+}$form and double dot lines - of the ionomers in protonated state. 


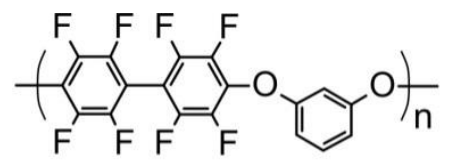

$\mathrm{P} 0-1.4$

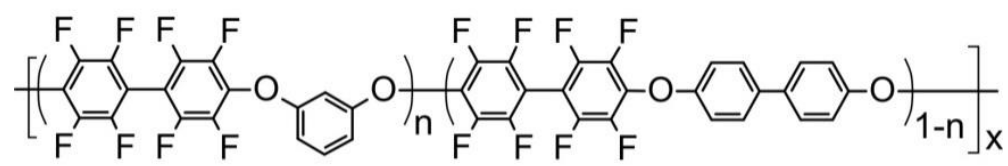

$\mathrm{P} 0-1.0$

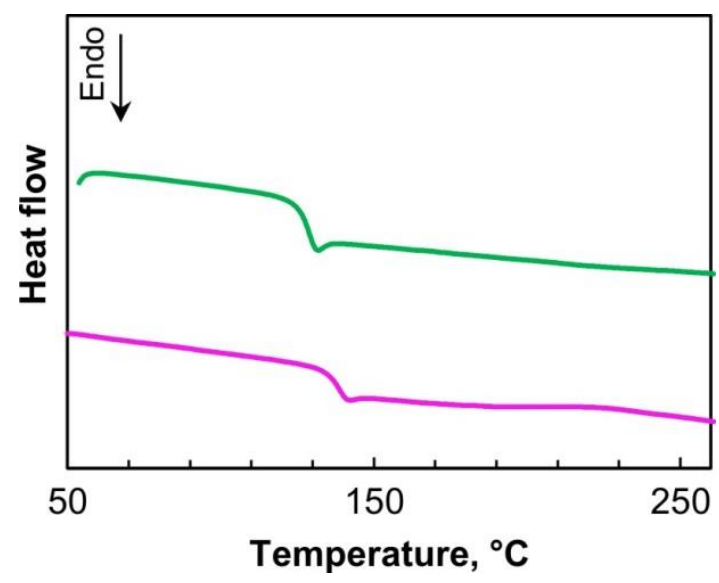

Figure SI2. Chemical structures and DSC thermograms of the ionomer backbones containing no perfluorosulfonic acid functions: green line is P0-1.4 - a backbone of the ionomer P1-1.4, and magenta thermogram is P0-1.0 - a backbone of P1-1.0.

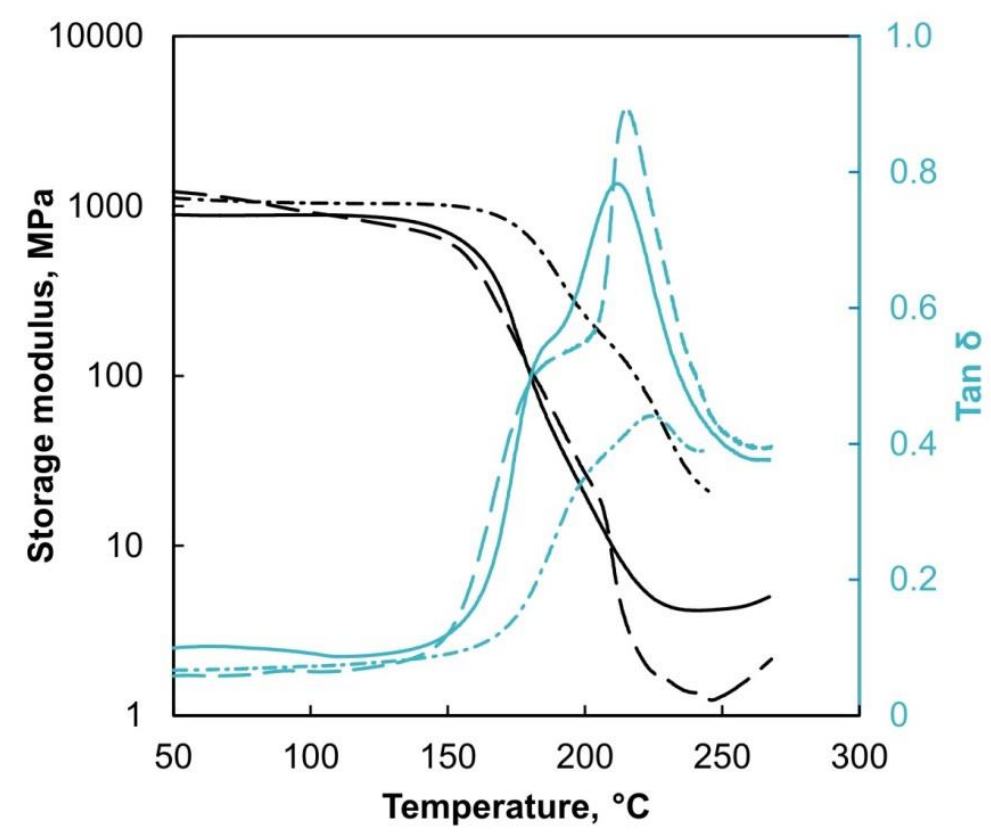

Figure SI3. DMA profiles of the P1 ionomers in their Li+ form: P1-1.0 - solid line, P1-1.2 - dashed line, P11.4 - dot-and-dash line. 


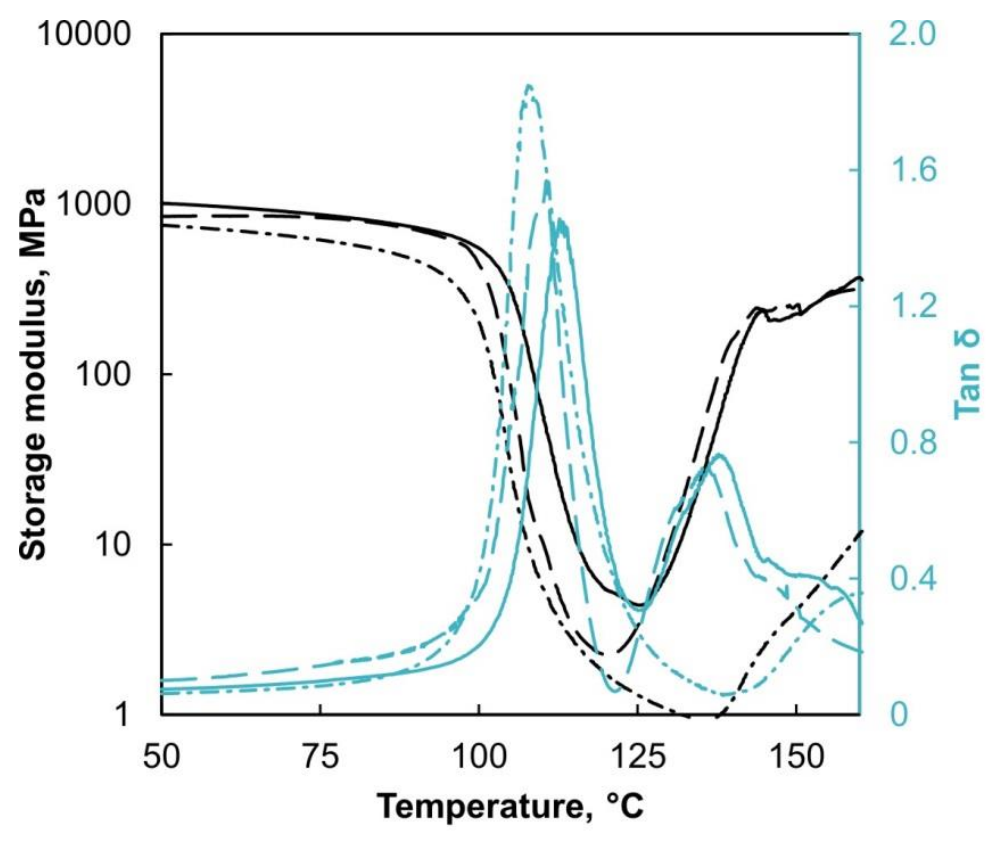

Figure SI4. DMA profiles of the P1 ionomers in their acidic form: P1-1.0 - solid line, P1-1.2 - dashed line, P1-1.4 - dot-and-dash line.

Table SI3. Hydration values for P1-1.4 membranes

\begin{tabular}{|c|c|c|c|c|c|c|}
\hline \multirow{2}{*}{$\begin{array}{c}\text { Temperature, } \\
{ }^{\circ} \mathrm{C}\end{array}$} & \multicolumn{6}{|c|}{$\lambda, \mathrm{H}_{2} \mathrm{O} / \mathrm{SO}_{3} \mathrm{H}$} \\
\hline & $\begin{array}{c}\mathrm{P} 1-1.0 \\
\mathrm{LiH}\end{array}$ & $\begin{array}{c}\mathrm{P} 1-1.0 \\
\mathrm{KH}\end{array}$ & $\begin{array}{c}\mathrm{P} 1-1.2 \\
\mathrm{LiH}\end{array}$ & $\begin{array}{c}\text { P1-1.2 } \\
\text { KH }\end{array}$ & $\begin{array}{c}\text { P1-1.4 } \\
\text { LiH }\end{array}$ & $\begin{array}{c}\text { P1-1.4 } \\
\text { KH }\end{array}$ \\
\hline 30 & \multicolumn{2}{|c|}{9} & \multicolumn{2}{|c|}{14} & 20 & 18 \\
\hline 40 & \multicolumn{2}{|c|}{10} & \multicolumn{2}{|c|}{14} & 22 & 21 \\
\hline 50 & \multicolumn{2}{|c|}{11} & \multicolumn{2}{|c|}{16} & 28 & 31 \\
\hline 60 & \multicolumn{2}{|c|}{12} & \multicolumn{2}{|c|}{19} & 48 & 48 \\
\hline 70 & \multicolumn{2}{|c|}{13} & \multicolumn{2}{|c|}{28} & \multicolumn{2}{|c|}{-} \\
\hline 80 & \multicolumn{2}{|c|}{17} & \multicolumn{2}{|c|}{51} & \multicolumn{2}{|c|}{ - } \\
\hline 90 & \multicolumn{2}{|c|}{23} & \multicolumn{2}{|c|}{ - } & \multicolumn{2}{|c|}{-} \\
\hline
\end{tabular}




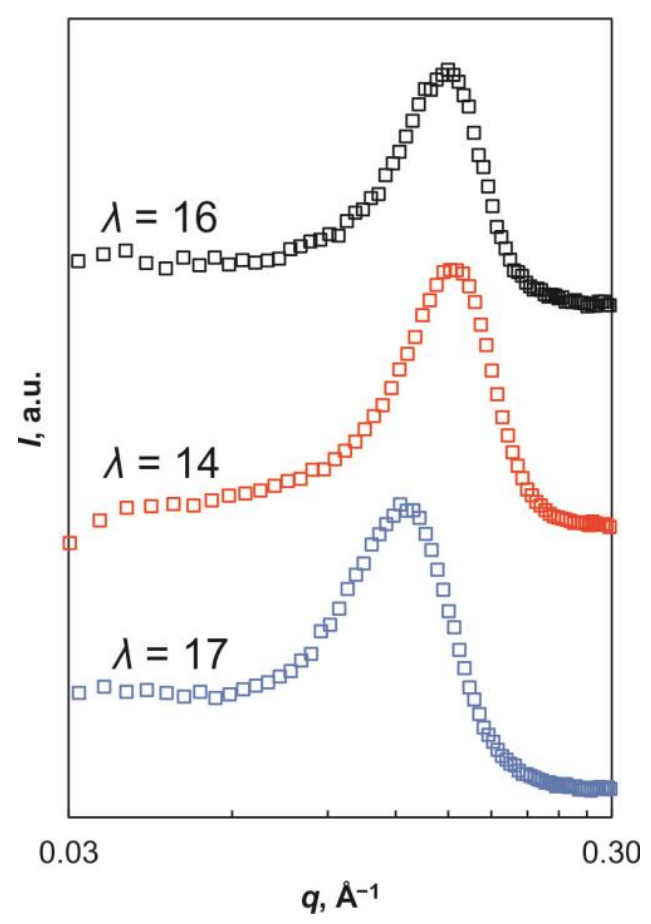

Figure SI5. SANS spectra of $\mathrm{P} 1 \mathrm{LiH}$ membranes, containing similar amount of water molecules in the structures (hydration numbers are attributed to each spectrum): P1-1.0 - blue down spectrum, P1-1.2 - red middle spectrum, P1-1.4 - black upper spectrum.

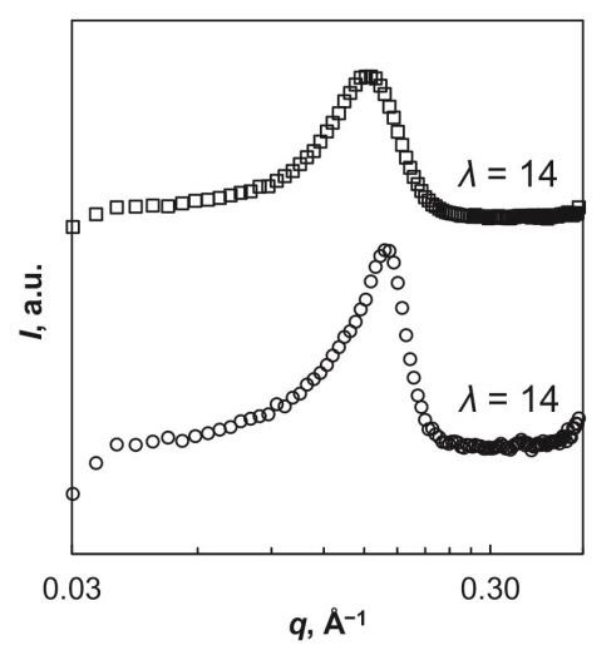

Figure SI6. SANS spectra of (口) P1-1.2 LiH and (०) P1-1.2 KH, when humidified at $40{ }^{\circ} \mathrm{C}$ in water ( $\lambda$-values of adsorbed water molecules per acidic site are attributed to each spectrum). 